\title{
PATTERN CONTROL IN A COUPLED CHAOTIC NETWORK AND ITS POSSIBLE APPLICATION IN COMMUNICATIONS
}

\author{
Yoshifumi NISHIO \\ Akio USHIDA \\ Tokushima University, 2-1, Minami-Josanjima, Tokushima 770, JAPAN \\ nishio@ee.tokushima-u.ac.jp
}

\begin{abstract}
In this study a controlling method of generated patterns in a simple coupled chaotic network is discussed. By adding an connection between two subcircuits, the number of generating patterns is restricted. Further, synchronization phenomenon of simple transmitter-receiver systems consisting of two chaotic networks is also examined.
\end{abstract}

\section{INTRODUCTION}

In our previous study [1][2], we have proposed a simple coupled chaotic network whose structural element is chaotic autonomous circuit. By computer simulations, we investigated the generation of interesting irregular self-switching phenomenon of a large number of spatial patterns characterized by both of attractors' shapes and phase difference of quasi-synchronization of chaos [3]. We found that 5632 different spatial patterns appear for the size of 3 by 3 and that switching speed could be controlled by tuning circuit parameters.

In order to apply our chaotic network to engineering systems, it will be first step to control generating spatial patterns. If we can restrict the number of generating patterns, it will correspond to embed information in the network. Further, if the network can produce irregular self-switching phenomenon of the restricted number of patterns, it will correspond to dynamical search of memorized informations.

In this study, we investigate irregular self-switching phenomenon of spatial patterns generated in a coupled chaotic network with an additional connection between two chaotic subcircuits. By computer simulations we found that we can successfully restrict the number of generating spatial patterns. It is also found that the number of restrained patterns are changed by the location of the additional connection. Further, we consider a transmitter-receiver system consisting of two chaotic networks. We will discuss about synchronization of irregular self-switching of spatial patterns.

\section{CIRCUIT MODEL}

In the original chaotic network without additional connection [1][2] can generate 11 different spatial patterns with respect to the phase states. (In the Refs. four patterns could not be found.) Actually, there coexist $2^{9}=512$ patterns with respect to the attractors' shapes. Hence, total number of the different spatial patterns is $512 \times 11=5632$. However, in order to concentrate on the control of the phase states, we ignore the switching of attractors' shapes throughout this article. The 11 spatial patterns are described as follows.

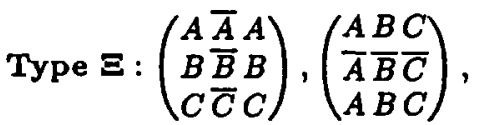

$$
\begin{aligned}
& \text { Type X:( } \left.\begin{array}{lll}
\frac{A}{B} & B & A \\
A & \bar{B} & \bar{B} \\
A & B
\end{array}\right)
\end{aligned}
$$

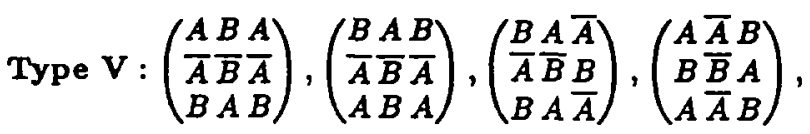

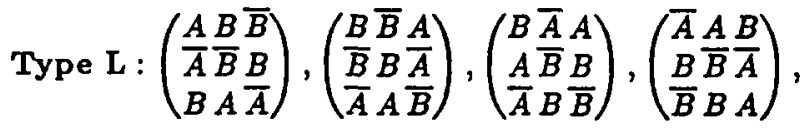

where different characters represent independent phase and $\bar{A}$ means the opposite phase of $A$. These patterns are generated by reason of the underlying physical mechanism; the network tends to minimize the currents through the coupling resistors $R$ s.

In this study we try to add a connection between two chaotic subcircuits for the purpose of the restriction of the number of generating patterns. An example of coupled chaotic networks with an additional connection is shown in Fig. 1. In the case, a connection is added from subcircuit 1 to 6 . In the followings we use simple drawings as (a) to represent such additional connections.

\section{RESTRICTION OF GENERATING PATTERNS}

There exist many conbinations of additional connections even for the network with the size of 3 by 3 . Then, we consider the case that the number of connections is one. Further, we ignore some connections which become equivalent after rotation of the network.

After the above restrictions, we can count up all connections. First connection is $0 \pm$. In this case, only one spatial pattern, modified Type $\Xi:\left(\begin{array}{l}A A B \\ \left.\bar{A} \bar{A} \frac{B}{A}\right) \\ A A B\end{array}\right)$, is observed.

Fig. 2(a) shows the time series of the Poincaré map obtained from the network with the above connection. From the figure we can see that no self-switchings occur. 


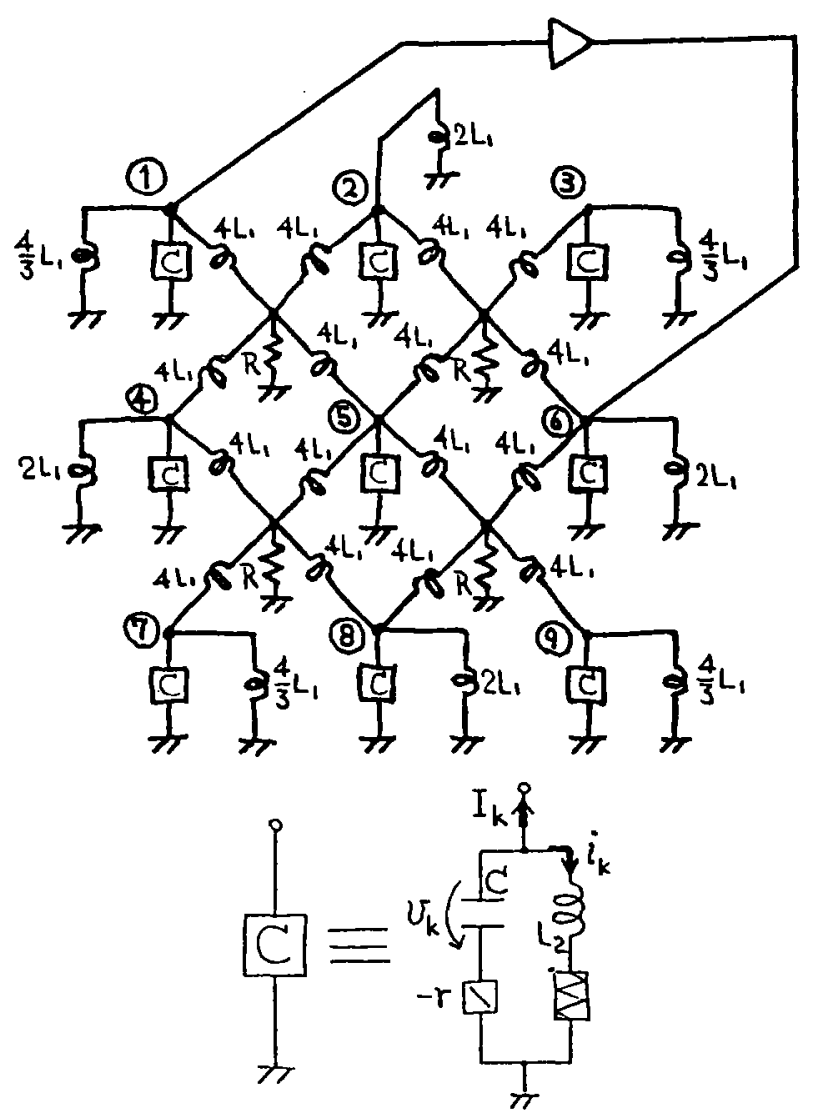

Fig. 1 Coupled chaotic network with a connection from subcircuit 1 to 6 .

Other connections and the corresponding spatial patterns are listed as

$$
\begin{aligned}
& \text { (1) } \rightarrow \text { (3) }:\left(\begin{array}{lll}
A & \bar{A} & A \\
B & \bar{B} & B \\
C & \bar{C} & C
\end{array}\right),\left(\begin{array}{lll}
A & B & A \\
\bar{A} & \bar{B} & \bar{A} \\
A & B & A
\end{array}\right),\left(\begin{array}{lll}
A & B & A \\
\bar{B} & \bar{A} & \bar{B} \\
A & B & A
\end{array}\right), \\
& \left(\begin{array}{lll}
\frac{A}{A} & \frac{B}{B} & \frac{A}{A} \\
B & A & B
\end{array}\right),\left(\begin{array}{lll}
\frac{B}{A} & \frac{A}{B} & \frac{B}{A} \\
A & B & A
\end{array}\right) \text {. } \\
& \text { Q }
\end{aligned}
$$

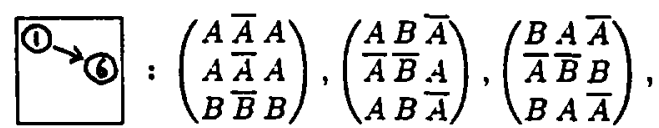

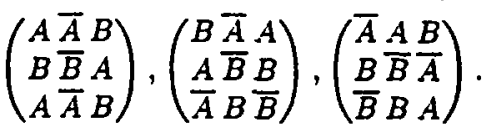

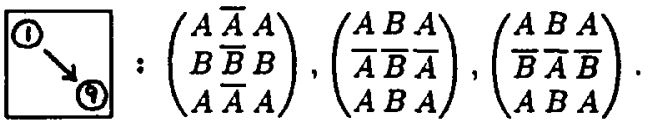

$$
\begin{aligned}
& \text { (3) }:\left(\begin{array}{lll}
A & \bar{A} & A \\
A & \bar{A} & A \\
B & \bar{B} & B
\end{array}\right) \text {. }
\end{aligned}
$$

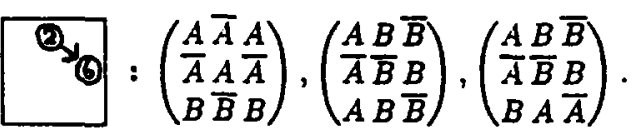
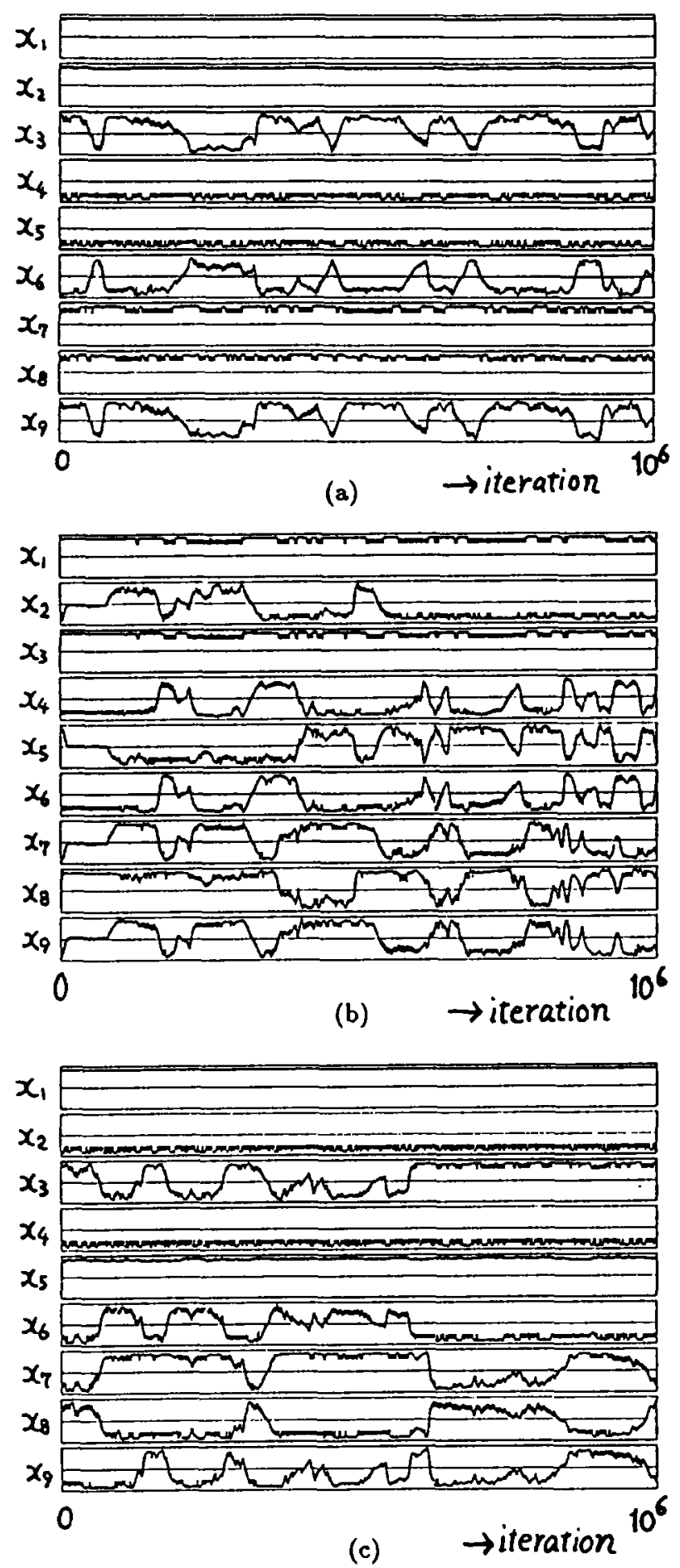

Fig. 2 Time series of Poincaré map. (a) Additional connection from 1 to 2 . (b) Additional connection from 1 to 3. (c) Additional connection from 1 to 5 . 


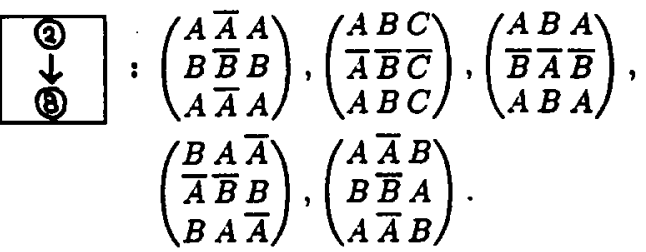

Figs. 2(b) and (c) show the time series of the Poincaré map obtained from the network with the connections (1) $\rightarrow$ (9) and $q_{(3)}$, respectively. From the figure we

can see that only some spatial patterns descrived above generate. By conbining some connections it would be possible to construct networks in which only some desired spatial patterns can be appear.

\section{TRANSMITTER-RECEIVER SYSTEM}

In this section we consider simple transmitter-receiver systems consisting of two chaotic networks. The simplest example is shown in Fig. 3. This system can be represented as

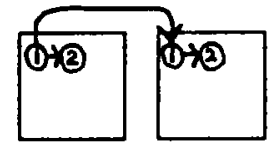

From this system we can observe chaos

synchronizations shown in Fig. 4. Time series of Poincaré map is also shown in Fig. 5. Because each subnetwork with this connection can produce only one spatial pattern, this result is not so interesting. However, this results indicate the possibility of synchronization of coupled chaotic networks.

Next, we consider the system of $\$$. this case each subnetwork can produce 5 different spatial patterns. Time series of Poincare map is shown in Fig. 6. From the figure we can see that $x_{1}, x_{3}, x_{1}^{\prime}$ and $x_{3}^{\prime}$ are synchronized. However, self-switching of spatial patterns are completely independent. In order to develop a new type of communication systems using chaos, we wish to exploit irregular self-switching phenomenon generated in coupled chaotic networks. Thus, we consider to transmit two

chaotic signals to the receiver side as

In this case, when the phase state of the transmitter side is $\left(\begin{array}{lll}A & \bar{A} & A \\ B & \bar{B} & B \\ C & \bar{C} & C\end{array}\right),\left(\begin{array}{lll}\frac{A}{B} & B & A \\ A & \bar{B} \\ A & B & A\end{array}\right)$ or $\left(\begin{array}{lll}\frac{B}{A} & \frac{A}{B} & \bar{A} \\ A & B & A\end{array}\right)$, the phese state of the receiver side must be also one of these. While when the phase state of the transmitter side is $\left(\begin{array}{c}\frac{A}{A} \bar{B} \frac{A}{A} \\ A B A\end{array}\right)$ or

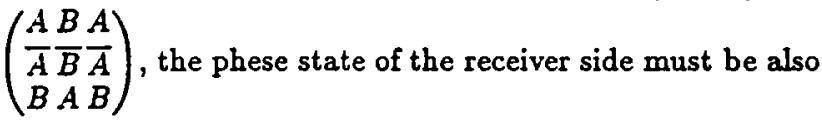
one of the two. Namely, spatial patterns of two subnetworks are not the very same. However, some of self-switchings at the transmitter side will transferred to the receiver side and only some chaotic subcircuits are synchronized by transmitted signals. Detailed results will be introduced in the presentation. Now, we do not know whether we can develop novel communication systems using our coupled chaotic network which can overcome conventional chaos communication systems. However, we hope that our system would contribute to develop novel types of communication systems.

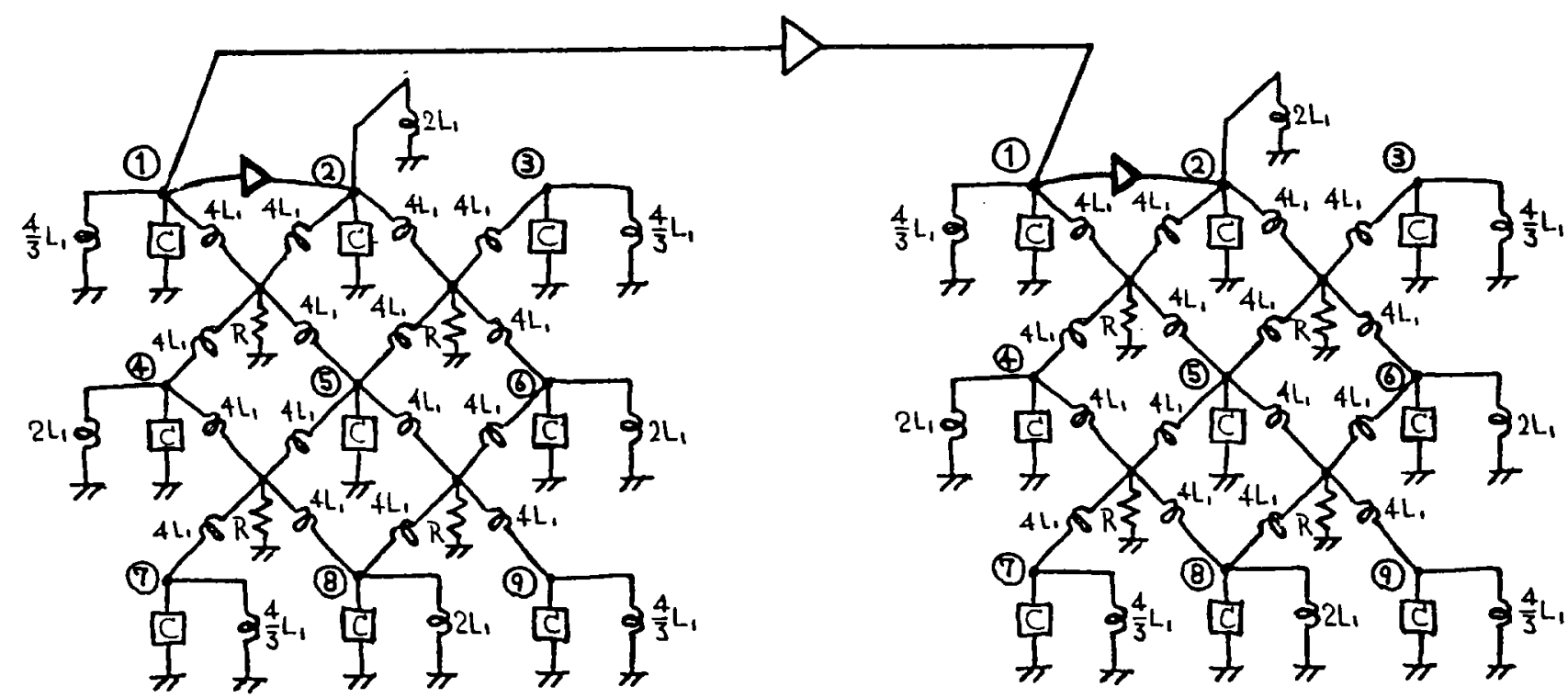

TRANSMITTER

\section{RECEIVER}

Fig. 3 Transmitter-receiver system using coupled chaotic networks. 


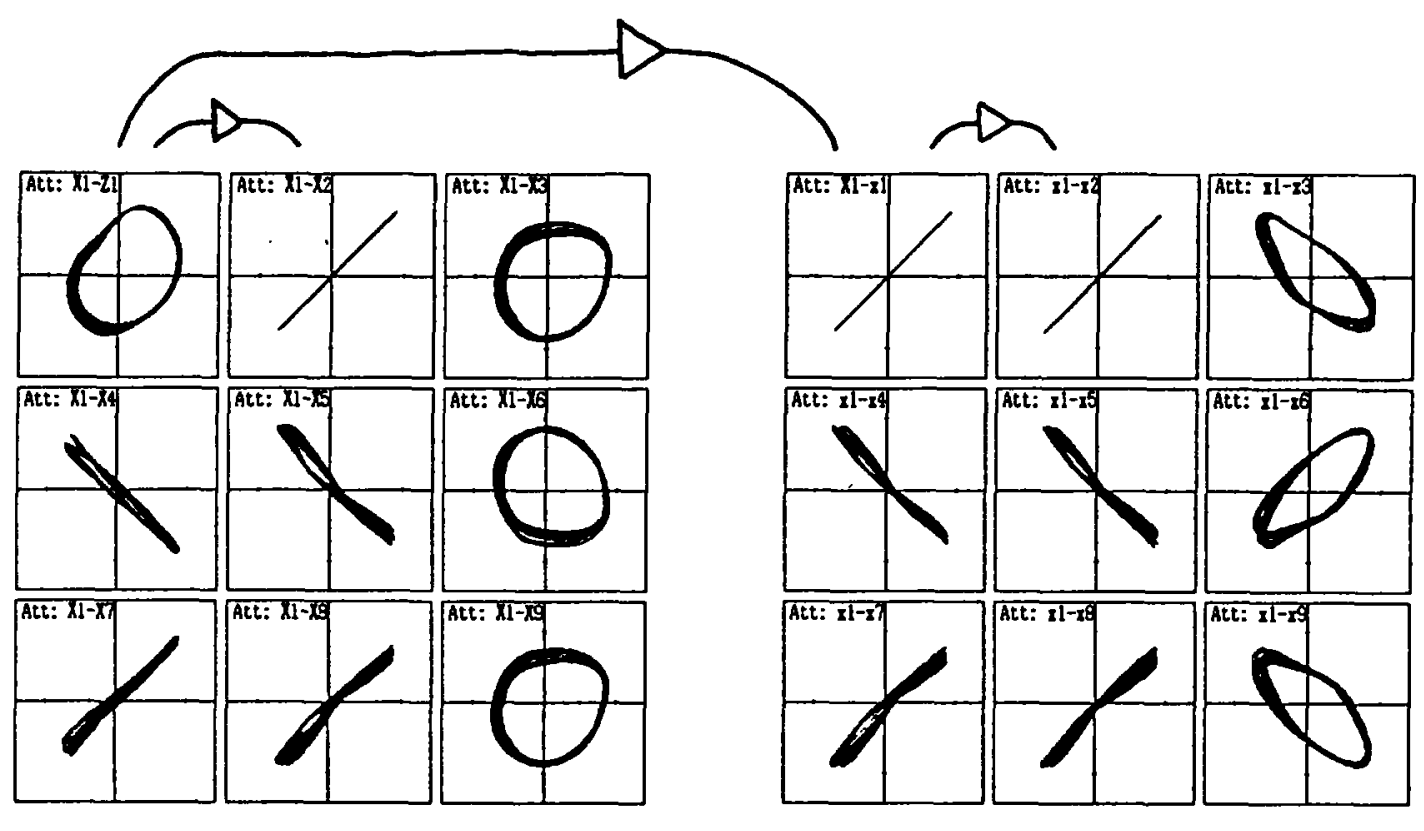

TRANSMITTER

RECEIVER

Fig. 4 Chaos synchronization in transmitter-receiver system.

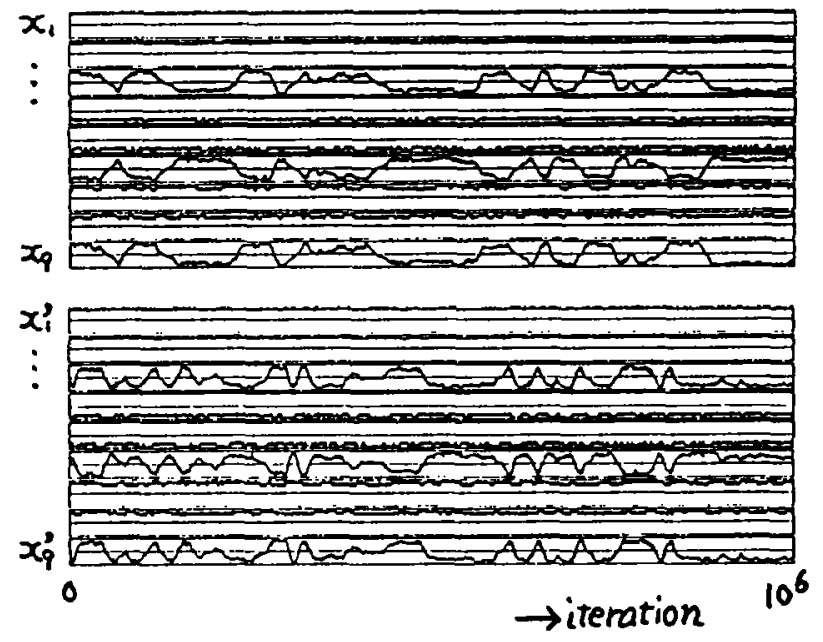

Fig. 5 Time series of Poincaré map. Connections are $x_{1} \rightarrow x_{2}, x_{1}^{\prime} \rightarrow x_{2}^{\prime}$ and $x_{1} \rightarrow x_{1}^{\prime}$.

\section{CONCLUSIONS}

In this study a controlling method of generated patterns in a simple coupled chaotic network has been discussed. By adding an connection between two subcircuits, the number of generating patterns was been successfully restricted. Further, synchronization phenomenon of simple transmitterreceiver systems consisting of two chaotic networks was also examined.

Our future research is to find attractive applications using synchronization of two coupled chaotic networks and to investigate larger size of coupled chaotic networks.
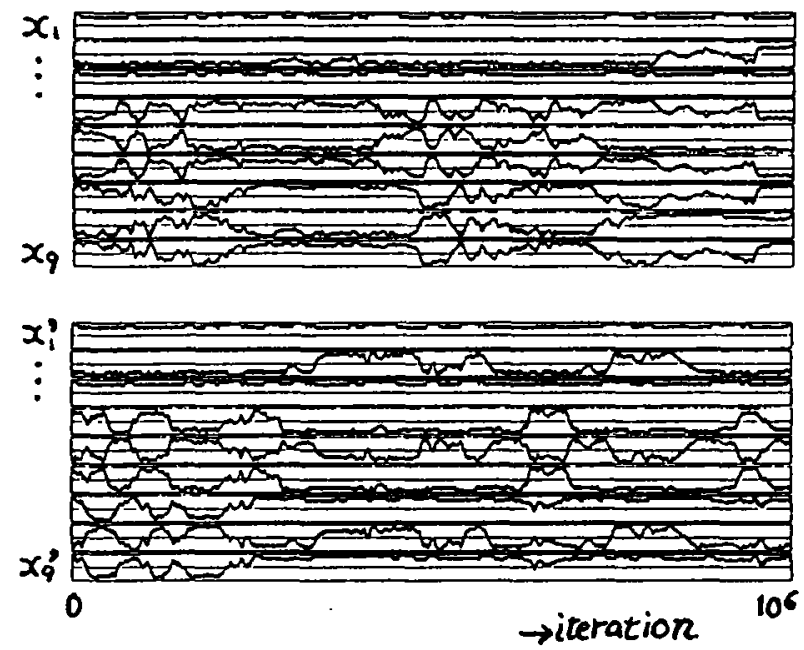

Fig. 6 Time series of Poincaré map. Connections are $x_{1} \rightarrow x_{3}, x_{1}^{\prime} \rightarrow x_{3}^{\prime}$ and $x_{1} \rightarrow x_{1}^{\prime}$.

\section{REFERENCES}

[1] Y. Nishio and A. Ushida, "Spatio-Temporal Chaos in Simple Coupled Chaotic Circuits," IEEE Trans. on Circuits Syst. I, vol. 42, no. 10, pp. 678-686, Oct. 1995.

[2] Y. Nishio and A. Ushida, "On Synchronization Phenomena in Coupled Chaotic Circuits Networks," Proc. ISCAS'96, vol. 3, pp. 92-95, May 1996.

[3] Y. Nishio and A. Ushida, "Quasi-Synchronization Phenomena in Chaotic Circuits Coupled by One Resistor," IEEE Trans. on Circuits Syst. I, vol. 43, no. 6, pp. 491496, Jun. 1996. 